University of Nebraska - Lincoln

DigitalCommons@University of Nebraska - Lincoln

$1-15-2012$

\title{
Discernible but limited introgression has occurred where Trichinella nativa and the T6 genotype occur in sympatry
}

Detiger B. Dunams-Morel

Mason V. Reichard

Luigi Torretti

Dante S. Zarlenga

Benjamin Rosenthal

Follow this and additional works at: https://digitalcommons.unl.edu/usdaarsfacpub

This Article is brought to you for free and open access by the U.S. Department of Agriculture: Agricultural Research Service, Lincoln, Nebraska at DigitalCommons@University of Nebraska - Lincoln. It has been accepted for inclusion in Publications from USDA-ARS / UNL Faculty by an authorized administrator of DigitalCommons@University of Nebraska - Lincoln. 


\title{
Discernible but limited introgression has occurred where Trichinella nativa and the T6 genotype occur in sympatry
}

\author{
Detiger B. Dunams-Morel ${ }^{\mathrm{a}}$, Mason V. Reichard ${ }^{\mathrm{b}}$, Luigi Torretti ${ }^{\mathrm{c}, 1}$, Dante S. Zarlenga ${ }^{\mathrm{a}}$, \\ Benjamin M. Rosenthal ${ }^{\mathrm{a}, *}$ \\ ${ }^{a}$ Animal Parasitic Disease Laboratory, Agricultural Research Service, USDA, 10300 Baltimore Avenue, Beltsville, MD 20705, United States \\ ${ }^{\mathrm{b}}$ Center for Veterinary Health Sciences, Oklahoma State University, Stillwater, OK 74878, United States \\ ${ }^{\mathrm{c}}$ Department of Environment, Government of Nunavut, Kugluktuk, Nunavut, Canada
}

\section{A R T I C L E I N F O}

\section{Article history:}

Received 23 November 2011

Received in revised form 4 January 2012

Accepted 5 January 2012

Available online 15 January 2012

\section{Keywords:}

Trichinella

Introgression

Trichinellosis

Zoonosis

Microsatellites

\begin{abstract}
A B S T R A C T
The genetic diversity within and among parasite populations provides clues to their evolutionary history. Here, we sought to determine whether mitochondrial and microsatellite DNA variation could be used to evaluate the extent of differentiation, gene flow and historical reproductive isolation among the freeze resistant parasites Trichinella nativa and the Trichinella T6 genotype infecting wolverines (Gulo gulo) in Nunavut, Canada. To this end, we genotyped Trichinella isolates derived from the diaphragms of 39 wolverines from this locale to reference strains of T. nativa and the Trichinella T6 genotype. Results showed that among a subset of 13 isolates examined, individuals resembled T. nativa in their mitochondrial DNA, but resembled the Trichinella T6 genotype when assayed at expansion segment V and the internal transcribed spacer of the nuclear rDNA. To adjudicate among these conflicting diagnoses, we further characterized each isolate at several nuclear microsatellite loci and again compared these to data from reference strains. Statistical assignment established that the nuclear genomes of most Nunavut isolates corresponded to those of the Trichinella T6 genotype; however, two isolates corresponded to T. nativa, and one isolate exhibited equal similarity to both reference strains. Taken as a whole, the evidence suggests that these isolates derive from the T. nativa matrilineage, but that their nuclear genomes resemble individuals previously designated as Trichinella T6. Assuming distinct lineages, this argues for cross-hybridization among these genotypes. Although introgression has occurred, recognizable genetic distinctions persist. One possibility is that selection disfavors the survival of hybrid offspring in most instances. Alternatively, the recent disappearance of glacial barriers might have increased contact, and therefore introgression. Broader geographic sampling will be required to determine the extent to which hybridization occurs beyond this particular geographic focus.
\end{abstract}

Published by Elsevier B.V.

\section{Introduction}

Human trichinellosis is contracted by ingesting uncooked meat containing encysted larvae of Trichinella spp. Although diagnosis can be difficult owing the variability of its clinical presentation and their resemblance to other pathological processes, common symptoms (in the acute phase, during larval migration from the gut to muscle tissues) often include gastroenteritis, abdominal pain, myalgia, and eosinophilia, tissue edema, vasculitis, and intravascular thrombi (Gottstein et al., 2009). These parasites infect a variety of mammalian, avian, and reptilian omnivores and carnivores (Pozio et al., 2009). Although most human infections are acquired by eating pork infected with Trichinella spiralis, wild game

\footnotetext{
* Corresponding author. Tel.: +1 301504 5408; fax: +1 3015048979.

E-mail address: Benjamin.Rosenthal@ars.usda.gov (B.M. Rosenthal).

1 Present address: Kitikmeot Inuit Association, Nunavut, Canada.
}

can represent an important source of food borne risk, especially in communities, such as those in the Canadian Arctic, where wild game is frequently consumed (Eaton, 1979; MacLean et al., 1989, 1992; Kapel, 1997; Serhir et al., 2001).

Two distinct taxa of Trichinella have been identified as endemic to the Canadian Arctic: Trichinella nativa and the Trichinella T6 genotype (hereafter referred to simply as 'T6'). Phylogenetic analysis attests to an especially close relationship between these genotypes, which share similar genetic and biological characteristics such as freeze resistance (Pozio and Zarlenga, 2005). Natural hybrids between these taxa have been identified (La Rosa et al., 2003) and larvae endemic to the Arctic are freeze resistant and capable of surviving prolonged periods within the frozen tissues of carnivores (Pozio et al., 1992; Kapel et al., 1995, 1999; La Rosa et al., 2003; Malakauskas and Kapel, 2003; Davidson et al., 2008; Reichard et al., 2008; Gajadhar and Forbes, 2010). Because only molecular and biochemical methods can be used to differentiate 




Possible Types of Natural Hybrids:



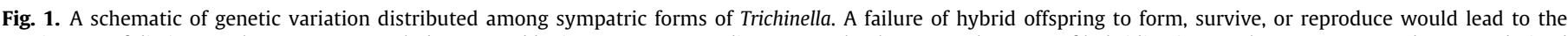

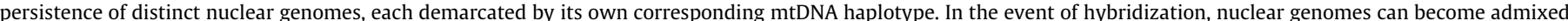

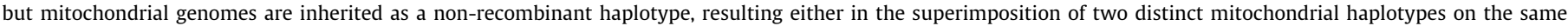
background of nuclear variation (if hybridization is rare and back-crosses are common), or resulting in the erosion of previous distinctions between nuclear lineages.

it from T. nativa, the T6 genotype has not yet been recognized as a distinct species (Pozio et al., 1992; Zarlenga et al., 1999, 2001; La Rosa et al., 2003; Reichard et al., 2008). Experimental studies have shown that the F1 progeny of a cross between a female ascribed to T. nativa and a male ascribed to T6 are infertile (La Rosa et al., 2003). Recently, a study of 41 wolverines (Gulo gulo) from Canada employed a diagnostic assay based on variation in nuclear rDNA (Reichard et al., 2008) and diagnosed 33 wolverines with T6 alone, two with both T. nativa and T6, and one with T. nativa alone.

In principal, contact between genetically-differentiated, sympatric lineages such as T. nativa and T6 could result in any of several outcomes (see Fig. 1): sympatric species could remain entirely reproductively isolated and maintain distinct nuclear and mitochondrial genes; rare introgression and frequent back-crossing could result in the transmission of only one mitochondrial haplotype to hybrid offspring that retain the other lineage's nuclear genes, or; frequent hybridization could erode distinctions between the nuclear genomes, compromising the capacity of any single marker to differentiate between them.

Here, we sought to further examine the extent and nature of gene flow between these two parasite taxa in a locale of established sympatry. In particular, we characterized a suite of nuclear microsatellite ( $\mu \mathrm{sat}$ ) markers and a portion of their mitochondrial genomes to assess whether parasites from these wolverines in Nunavut, Canada indeed comprise two, reproductively independent populations differentiable by specified genetic attributes, or instead demonstrate evidence of introgression.

\section{Materials and methods}

\subsection{Sample collection, isolation, and DNA extraction}

We employed previously described methods to characterize variable genetic markers from pools of larvae digested from infected animals, and also modified these procedures to verify their performance on individual larvae. As described below, our analyses probed genetic information from 39 pools of larvae, each from a different wolverine, and 18 individual larvae (three from each of six wolverines). Among these individual larval DNAs, six were chosen for whole genome amplification (WGA).

First, muscle larvae of Trichinella spp. were recovered from 39 wolverines in the Nunavut, Canada between November 2006 and April 2007 (Table 1 and Fig. 3) by pepsin: $\mathrm{HCl}$ digestion of the diaphragm, tongue, masseter, quadriceps, and deltoids. Separate pools of larvae obtained from each animal were stored in vials containing $70 \%$ ethanol for 3 years prior to commencement of this study. DNA was extracted using the DNAeasy protocol (Qiagen Corp.) after decanting most of the ethanol from each vial and evaporating residual storage fluid by means of vacuum centrifugation for $30 \mathrm{~min}$.

DNA was also extracted from three individual larvae from each of six isolate pools (KU 5, 7, 10, 13, 20, and 33; see Table 1), using the DNA IQ System Tissue \& Hair Extraction Kit (Promega Corp.) according to manufacturer recommendations. Of these three larval extracts, the one producing the strongest amplification product in an initial PCR screen using the TP19 $\mu$ sat locus (Rosenthal et al., 2008) was selected to undergo WGA using the GenomePlex Single Cell Whole Genome Kit (Sigma-Aldrich) in order to determine whether this procedure would increase the number of loci from which microsatellites could be called from larvae that had undergone prolonged storage in ethanol. For WGA, $2 \mu \mathrm{l}$ of the primary extract was diluted 5 -fold, $9 \mu \mathrm{l}$ of which was subsequently used as template. Of the $75 \mu \mathrm{l}$ total, $15 \mu \mathrm{l}$ of this WGA reaction were purified using Qiaquick PCR purification kit (Qiagen Corp.). The final elution was performed with $50 \mu$ l of the kit's elution buffer.

\subsection{Genetic analyses}

\subsubsection{Multiplex assay for variation in nuclear ITS-2}

PCR amplification of the expansion segment V (ESV) and the internal transcribed spacer 1 (ITS-1) of the nuclear large subunit rDNA (LSU rDNA) was performed on 13 larvae derived from wolverines in Nunavut, as well as on reference isolates of $T$. nativa and T6 (listed in Table 1) using a previously described multiplex PCR assay (Zarlenga et al., 1999). These products were separated 
Table 1

Comparison of a subset of isolates characterized at by all three genotyping methods (multiplex, microsatellite, and mitochondrial DNA).

\begin{tabular}{|c|c|c|c|c|c|c|c|}
\hline & \multirow[t]{2}{*}{ Isolate } & \multirow{2}{*}{$\begin{array}{l}\text { Reference } \\
\text { (IT Code) }\end{array}$} & \multirow[t]{2}{*}{ Country } & \multirow[t]{2}{*}{ Host } & \multicolumn{3}{|c|}{ Designation based on } \\
\hline & & & & & Multiplex & Microsattelite & mtDNA \\
\hline \multirow[t]{9}{*}{ Reference strains } & T. nativa EU090 & 70 & Russia & Wolf & T. nativa & T. nativa & T. nativa \\
\hline & T. nativa AS092 & 410 & Arctic & Polar bear & T. nativa & T. nativa & T. nativa \\
\hline & T. nativa EU093 & 558 & Finland & Raccoon dog & T. nativa & T. nativa & T. nativa \\
\hline & T. nativa NA095 & 43 & Canada & Polar bear & T. nativa & T. nativa & T. nativa \\
\hline & T. nativa NA096 & 45 & Canada & Fox & T. nativa & T. nativa & T. nativa \\
\hline & T6 & 40 & USA & Cougar & T6 & T6 & $\mathrm{T} 6$ \\
\hline & T6 & g73* & USA & Grizzly bear & $\mathrm{T} 6$ & T6 & T6 \\
\hline & $\mathrm{T} 6$ & g72 & USA & Fox & T6 & T6 & T6 \\
\hline & T6 & g343* & USA & Wolf & T6 & T6 & T6 \\
\hline \multirow[t]{7}{*}{ Pooled prior to msat genotyping } & KU 07 & 1876 & Canada & Wolverine & T6 & $\mathrm{T} 6$ & T6 \\
\hline & KU 08 & 1877 & Canada & Wolverine & T6 & T6 & T. nativa \\
\hline & KU 22 & 1891 & Canada & Wolverine & T6 & T6 & T. nativa \\
\hline & KU 24 & 1893 & Canada & Wolverine & T6 & $\mathrm{T} 6$ & T. nativa \\
\hline & KU 32 & 1900 & Canada & Wolverine & T6 & $\mathrm{T} 6$ & T. nativa \\
\hline & KU 35 & 1902 & Canada & Wolverine & T6 & T. nativa & T. nativa \\
\hline & KU 41 & 1905 & Canada & Wolverine & T6 & T6 & T. nativa \\
\hline \multirow[t]{6}{*}{ Genotyped individually in all assays } & KU 05 L1 & 1874 & Canada & Wolverine & T6 & $\mathrm{T} 6$ & T. nativa \\
\hline & KU 07 L1 & 1876 & Canada & Wolverine & T6 & $\mathrm{T} 6$ & T. nativa \\
\hline & KU 10 L1 & 1879 & Canada & Wolverine & $\mathrm{T} 6$ & $\mathrm{~T} 6$ & T. nativa \\
\hline & KU 13 L2 & 1882 & Canada & Wolverine & T6 & $\mathrm{T} 6$ & T. nativa \\
\hline & KU 20 L1 & 1889 & Canada & Wolverine & T6 & T6 & T. nativa \\
\hline & KU 33 L2 & 1898 & Canada & Wolverine & $\mathrm{T} 6$ & $\mathrm{~T} 6$ & T. nativa \\
\hline
\end{tabular}




systems.

ITRC ISS code not assigned for g72, g73, or g343.

and visualized using the QiAxcel electrophoresis system (Qiagen Corporation).

\subsubsection{Microsatellite assays}

To analyze the 39 extracts derived from pooled larvae using nine allelic $\mu$ sat markers, we employed methods that were previously used to genotype a large sample of Trichinella spp., including seven isolates of T. nativa and one isolate of the T6 genotype (Rosenthal et al., 2008). That study, which identified a notable deficit of variability in the T. spiralis endemic to Europe and the Americas, completely genotyped all eight aforementioned isolates of T. nativa and T6. Among those isolates, all loci were variable, ranging from 2 to 8 alleles/locus (mean 4.4, se 2.2), 17 of 72 (24\%) individual allele calls were heterozygous, and each of the eight isolates bore a unique multilocus genotype. To analyze these markers from extracts derived from individual larva, these methods were modified as follows: $2.5 \mu \mathrm{l}$ of template were used in 40 cycle PCRs. To assess the optimal volume of such products to be analyzed on an ABI 3730 capillary electrophoresis instrument, various volumes of the PCR products ( $2 \mu \mathrm{l}, 1 \mu \mathrm{l}$, and a $1 \mu \mathrm{l}$ of a 50 -fold dilution) were mixed with HiDi Formamide containing 0.75\% GeneScan 500 LIZ molecular weight standards (Applied Biosystems) to a total volume of $10 \mu \mathrm{l}$.

Newly sampled isolates were compared to each other, and to those samples previously reported by Rosenthal et al. (2008). In total, 168 isolates were compared at the nine loci. Structure (v 2.3.3) was used to assign individuals to any one of $K$ populations, using the admixture model of ancestry and using an initial Dirichlet parameter of 1.0, a uniform prior, and a maximum alpha value of 10.0. The SD for updating alpha was set at 0.025 . Allele frequencies were assumed to be correlated among populations with a mean prior Fst of 0.01 , a prior SD of Fst of 0.05 , a constant Lambda set initially at 1.0. We estimated the probability of the data under the model and used 10 as the frequency of Metropolis update for Q; $K$ was varied from 3 to 10 . Each run commenced with a burn in period of 100,000 and sampled from a posterior distribution composed of 1 million generations. The probability of the data under each model was evaluated 10 times, and $K=7$ was selected as a model that captured sufficient complexity without unduly inflating variance terms, using the method described by Yang et al. (2005).

\subsubsection{Mitochondrial DNA assays}

Approximately $1800 \mathrm{bps}$ of the mitochondrial genomes from seven isolate pools ( $\mathrm{KU} 7,8,22,24,32,35$, and 41), as well as templates derived from six individual larvae (KU 5L1, 7L1, 10L1, 13L2, 20L1, and 33L2) were sequenced, corresponding to the first half of the region employed in a previous study (Rosenthal et al., 2008). Similar procedures were applied to several reference isolates of $\mathrm{T} 6$ and $T$. nativa (Table 1 ) and a reference isolate of murrelli. The 1800 bps was generated from three amplified DNA fragments using the following primer combinations; Trich-cob-F1 (Lavrov and Brown, 2001) and Trich-cob-R3 (973 bp), Trich-seqF2 and Trich-seq-R4 (938 bp), or Trich-seq-F3 and Trich-seq-R5 (1027 bp) (Table 2). The same primers were used for DNA cycle sequencing. BLAST was employed to verify the resulting sequences as homologous to the target.

Matrilineal descent relationships were reconstructed from mitochondrial DNA sequences under the criterion of Minimum Evolution as implemented in MEGA v4.1 (Tamura et al., 2007; Kumar et al., 2008). Evolutionary distances were computed using the Maximum Composite Likelihood method and are presented as the number of base substitutions per site. All positions containing gaps and missing data were eliminated from the analysis,

Table 2

Primer sequences for amplifying and sequencing mitochondrial DNA.

\begin{tabular}{ll}
\hline Primer & Sequence \\
\hline Trich-cob-F1 & CAATCCATTAGGTACACACTCAC \\
Trich-seq-F2 & CACATGATTCACAATCACCT \\
Trich-seq-F3 & GCAGTAAGAAACCCATCAGA \\
Trich-seq-R3 & TAAGTAAGATTTCAATGGCG \\
Trich-seq-R4 & AAATCTTAAGTACTCGTAGTTTA \\
Trich-seq-R5 & AGGTTGTGATTATTAGTTTCTAGGG \\
\hline
\end{tabular}


resulting in a total of 1160 positions in the final dataset including product from individual and pooled larvae.

Microsatellite and multiplex markers as well as mitochondrial DNA sequences from all wolverine isolates were compared to known reference isolates of T. nativa, T6, and other congeners (Table 1, Fig. 3). To better contextualize the range of variation in microsatellite markers, the present sample was also compared to those isolates of other species of Trichinella previously analyzed Rosenthal et al. (2008).

\section{Results}

\subsection{Nuclear rDNA}

From each of the 13 larvae assayed from wolverines in Nunavut using the multiplex PCR assaying variation in nuclear rDNA (Zarlenga et al., 1999), two bands were amplified whose lengths corresponded to those amplified from reference isolates of the T6 genotype (Fig. 2). In previous assays of 10 other larvae digested from wolverine KU-35, some resulted in the amplification of two bands, whereas others resulted in the amplification of only one (Reichard et al., 2008).

\subsection{Microsatellite assays based on pooled larvae, individual larvae, and individual larvae subjected to whole genome amplification}

A scorable was achieved in 336/351 (96\%) attempts from the 39 extracts derived from pooled larvae (Fig. 3). Observed heterozygosity ranged among loci from $2 \%$ to $74 \%$ (mean $30 \%$, se $26 \%$ ). True heterozygosity may have been greater, if null alleles resulted in misclassifications of heterozygotes as homozygous. The contribution of such null alleles can be estimated from the frequency of 'double nulls' (15\% in this dataset); doing so, however, requires strong assumptions about the degree of inbreeding taking place (for example, null alleles would be estimated at a frequency of $39 \%$ if random mating were assumed; however, frequent inbreeding is expected for species of Trichinella because larval cohorts must often derive from synchronous exposure to closely related parents; La Rosa et al., in press).

Of the 18 extracts derived from individual larva, 14 were successfully amplified in an initial screen targeting $\mu$ sat locus 1 . Overall, the genotyping success of these individual larval extracts was poor (mean number of loci amplified $=4.28$, variance 1.64 ), perhaps because they had been stored for a considerable period in ethanol prior to DNA extraction. From 10 of these 14 larvae, genotyping was possible at 4 or more of the 9 attempted loci. Six of these 14 templates, each derived from a different wolverine, were subsequently chosen to undergo WGA. Doing so improved the number of $\mu$ sat loci amplified (mean $=7.2$, variance 2.16 ), rendered all six capable of being genotyped at 4 or more of the 9 loci, enabled two of the six to be completely genotyped, and enabled one other to be genotyped at 8 of the 9 loci.

\subsection{Population assignment and phylogenetic reconstruction based on variation in nuclear microsatellites}

Qualitative assessment of the distribution of microsatellite variation (Fig. 3) identifies notable and exclusive similarity between isolates from Nunavut and reference isolates of T6 (locus 5, 6, and 8). At other loci, discrimination is not possible owing to the fact that reference isolates are variable, encompassing alleles that are also observed in Nunavut samples $(1,2,4)$ or that are distinct from those most common in Nunavut samples (7, 9). At one locus (3) the same two alleles, in relatively equivalent proportions, occur in isolates from Nunavut and those of each reference taxon.

The data depicted in Fig. 3 were then subjected to statistical analysis to identify subdivisions among the isolates. On the basis of assaying alleles of nuclear microsatellites, nearly all isolates (larval pools) corresponded to reference isolates of T6 (depicted in dark blue, Fig. 4; identified in Table 1). This broadly agreed with a prior assessment of infections in these wolverines based on application of multiplex PCR to individual larva, wherein all infected wolverines but three were diagnosed with T6, exclusively (Reichard et al., 2008). By contrast, a small subset of isolates aligned with reference specimens of $T$. nativa that aggregated in a separate population (depicted in red, Fig. 4). In two cases (KU 02 and KU 35), wolverines in which larvae of T. nativa had been diagnosed on the basis multiplex analysis were assigned with high-level agreement to T. nativa when analyzed as pools with microsatellite alleles. However, the prior work had also identified, in these two wolverines, parasites corresponding to T6; their contribution was not evident from the analysis of microsatellite data. Pooled larvae from another wolverine (KU 40), that had previously been diagnosed with $T$. nativa alone, supported amplification of microsatellites assigned with $50 \%$ probability to T. nativa and $50 \%$ probability to T6. (These three cases are indicated by arrows in Fig. 4). Microsatellite genotyping of individual larvae resulted in exclusive assignment to $\mathrm{T} 6$ in all but two cases. In one such larva (KU $05 \mathrm{~L} 1$ ), the affinity to T6 was $80 \%$ whereas the affinity to $T$. nativa was $20 \%$. Thus, the present approach broadened the sampling of the nuclear genome, but (when applied to pools rather than individuals), failed to discern two cases of established admixture.

A neighbor-joining tree was reconstructed from a matrix of pairwise $\mathrm{dmu}^{2}$ distances (Goldstein et al., 1995) as implemented by Populations v.1.2.30 (Fig. 5). This analysis, which is sensitive to biases induced by missing data, was restricted to templates that were completely genotyped at all $\mu$ sat loci (including 32 larval pools and two individual larva derived from wolverines in Nunavut, as well as larval pools derived from four reference isolates of T6, seven reference isolates of T. nativa, and 43 reference isolates from outgroup taxa). This analysis generally allied isolates from these wolverines with reference isolates from T6. For some isolates (i.e. KU 24) this affinity was pronounced (represented by an especially short branch); less affinity (depicted by longer intervening branches) was recognized for others (including KU 02 and 35, identified above as resembling T. nativa as judged by Structure).

\subsection{Mitochondrial DNA sequencing}

Mitochondrial loci were consistently amplified from all templates, including those derived from individual larva that had not undergone WGA.

A subset of these (Fig. 6) were sequenced and compared over 1170 bps of mitochondrial DNA. These derived from seven pooled isolates and six individual larva infecting wolverines in Nunavut,





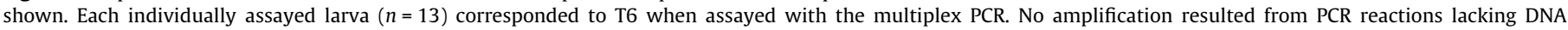
template. 


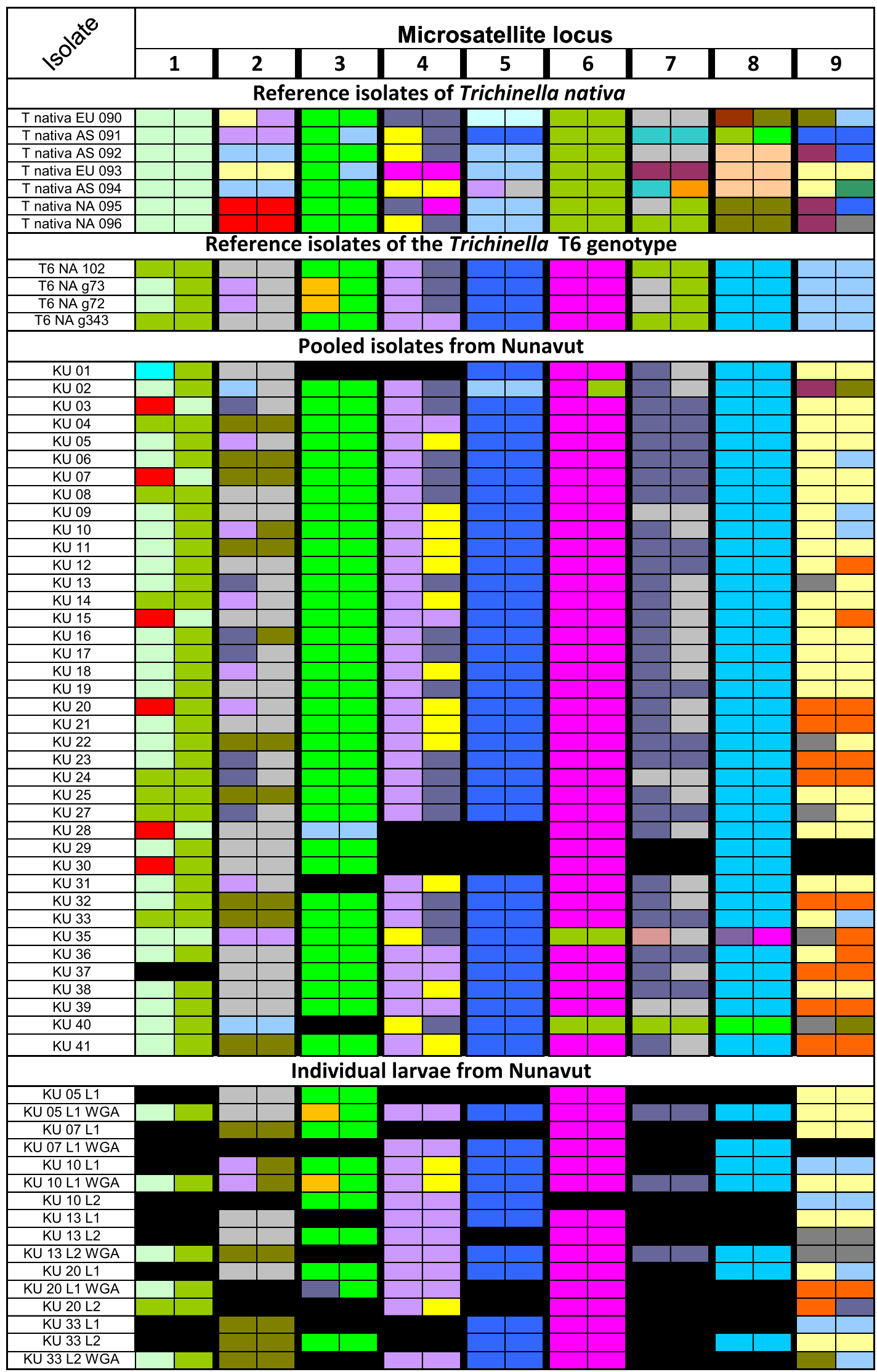

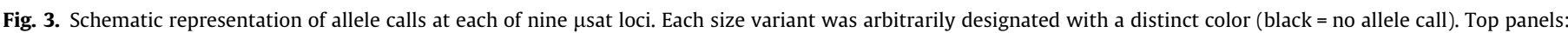

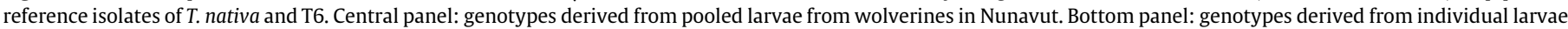

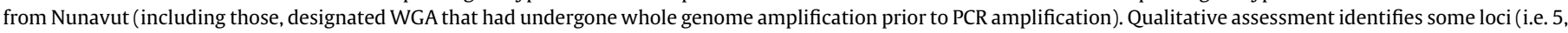



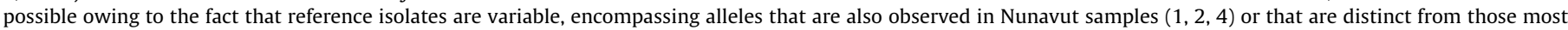
common in Nunavut samples $(7,9)$. At one locus (3) a particular allele is characteristic of Nunavut isolates and also those of each reference taxon. 


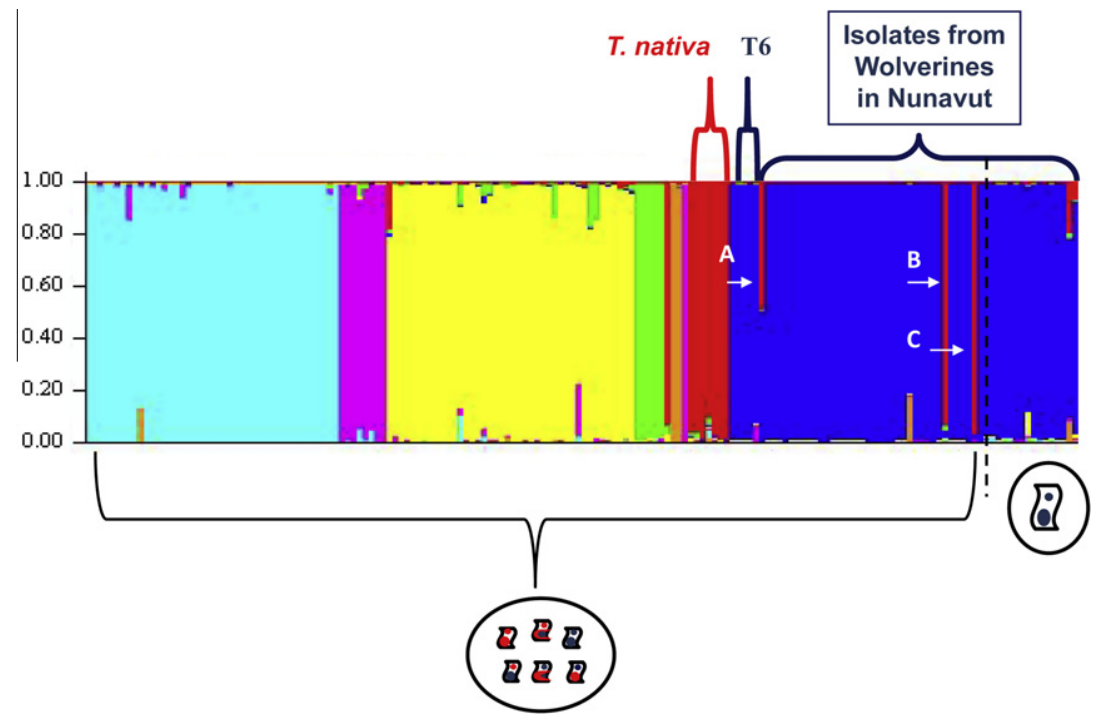

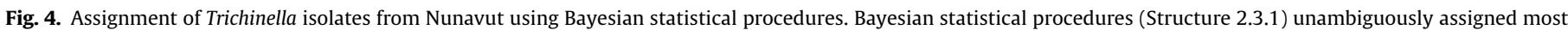

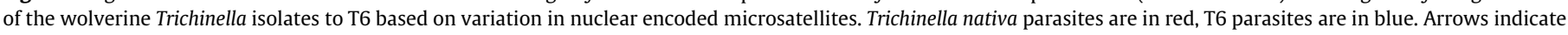



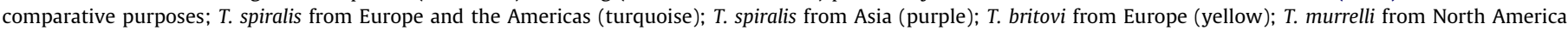

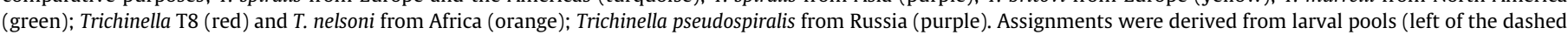
lines) or individual larvae (right of the dashed lines).

Canada. In addition, we sequenced 4 reference isolates from North American T6, and five isolates representing T. nativa derived from Arctic regions of Europe, Asia, and North America (Table 1) (GenBank Accessions JQ430659-JQ430674, JQ511986-JQ511990 and JQ513385). Doing so identified eight single nucleotide polymorphisms that segregated together, defining exactly two haplotypes. In every instance, each of the five reference isolates of T. nativa (Table 1, Fig. 6) bore the same haplotype; this haplotype was distinct from the haplotype shared by each of four reference isolates of T6 (Table 1, Fig. 6). From wolverines in Nunavut, all 13 sequenced templates (whether derived from pooled or individual larvae) corresponded perfectly to the haplotype ascribed to T. nativa. Phylogenetic reconstruction of variation in these sequences shows that a subset of Nunavut isolates and T. nativa share common maternal ancestry (Fig. 6).

\section{Discussion}

Previous reports have found wild carnivores infected with natural hybrids of T. nativa and T6 (Pozio et al., 2009).

The history of Trichinella spp. can be successfully interrogated by analyzing the extent and distribution of genetic variation. For example, the basic relationships and modes of diversification among parasites in the genus were inferred from such data (Zarlenga et al., 2006) and affinities in mitochondrial DNA and microsatellite loci substantiated the conclusion that $T$. spiralis was introduced to the Americas from Europe (Rosenthal et al., 2008). Here, we employed similar methods to address the genetic relationship between $T$. nativa and T6. In particular, we sought to determine the extent and nature of regional gene flow that may be occurring between these lineages where both types occur. Genotyping with mtDNA enabled us to trace the maternal ancestry of these isolates, whereas $\mu$ sat genotyping provided a means to assess whether interbreeding has eroded historical barriers to nuclear gene flow.

For over a decade, isolates of Trichinella have been discriminated by interrogating variation in the ITS and ESV of nuclear rDNA (Zarlenga et al., 1999). Using this assay, a common band is amplified from all freeze-resistant isolates. However, a second product, derived from the ITS- 1 , has been taken as specifically diagnostic for isolates of T6. The present study employed this method as an initial screen for identification, but expanded upon it given the insufficiency of any single locus to describe the extent and character of gene flow that may be occurring.

In each of the thirteen isolates from Nunavut tested here (Table 1), applying this standard molecular diagnostic tool resulted in the amplification of the two bands typical of T6 (Fig. 2). The multiplex PCR data generated herein coincided well with data presented elsewhere in diagnosing these worms as T6 (Reichard et al., 2008). Likewise, allelic variation in nuclear-encoded $\mu$ sat loci generally supported the assignment of parasites derived from these wolverines to T6; however, two isolates were assigned with near certainty to T. nativa, and a third isolate was assigned with $50 \%$ probability to T. nativa and $50 \%$ probability to T6 (Fig. 4).

A phylogenetic approach similarly aligned most of these isolates with T6 (Fig. 5). The established sister relationship between T. nativa and T6 (Zarlenga et al., 2006) was not recovered in this neighbor joining tree, perhaps because microsatellite variation quickly saturates over prolonged evolutionary intervals. However, all reference isolates of T. nativa and T6 did cluster into two discrete groups. In spite of the observation that nuclear markers generally allied parasites in our sample with reference isolates of the $\mathrm{T} 6$, the matrilineal descent of all 13 sequenced isolates corresponded perfectly with that of T. nativa. This was true when the source of the sequenced PCR product was either pooled or individual larvae.

Taken together, these data affirm the persistence of geneticallydifferentiated nuclear genomes and show that at least a subset of parasites in this region resembling $\mathrm{T} 6$ in their nuclear genomes nonetheless carry mtDNA inherited from a T. nativa maternal ancestor (akin to Scenario 2 depicted in Fig. 1). Thus, we can abandon as inapplicable the first scenario depicted in Fig. 1, wherein two sympatric lineages have maintained absolute genetic isolation in the vicinity of Nunavut. Clearly, hybridization has resulted in the transmission of one matrilineal haplotype to two distinct genomic backgrounds, akin to the second population scenario depicted in Fig. 1. This introgression of the T. nativa mtDNA haplotype is 


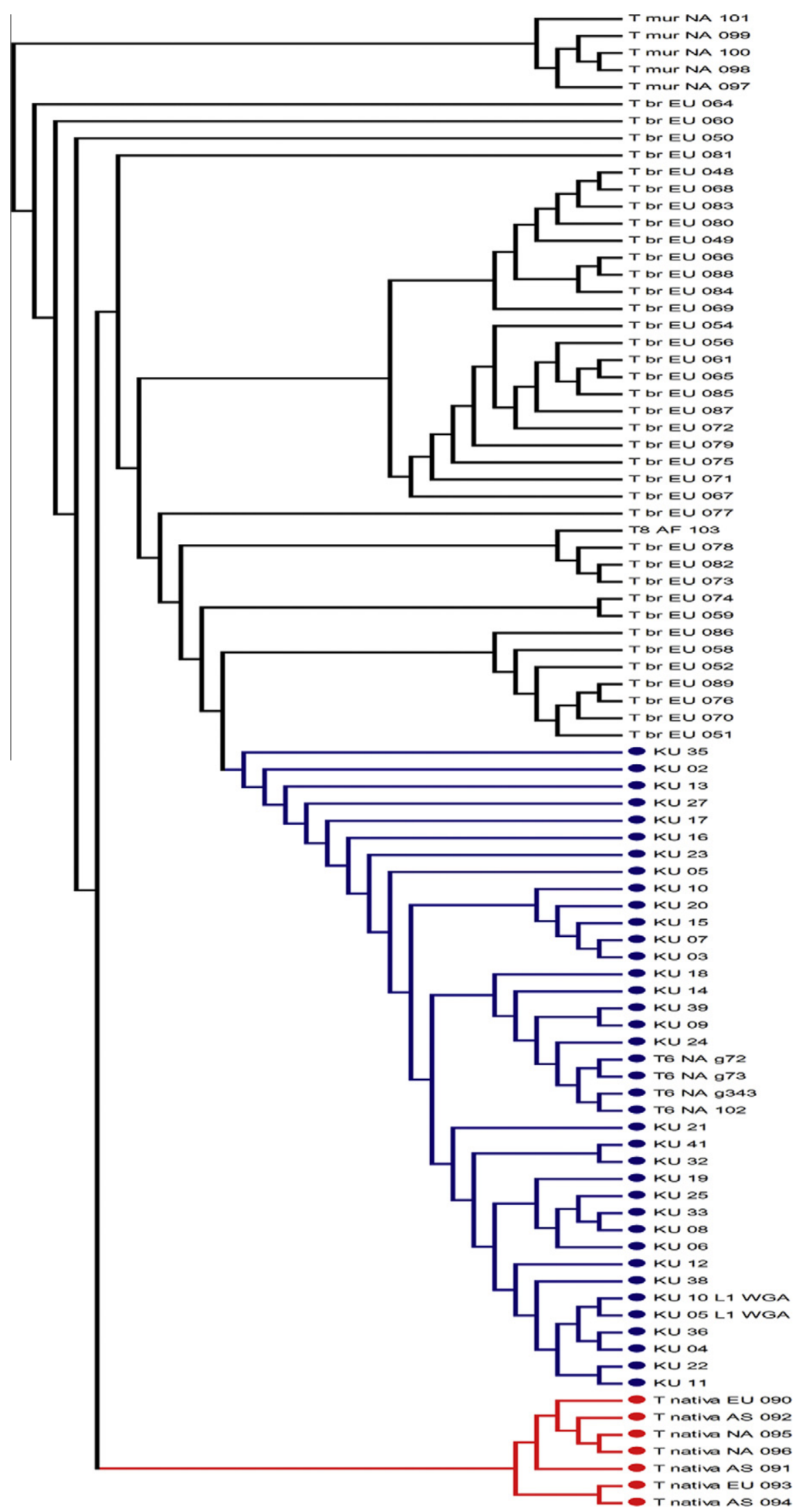



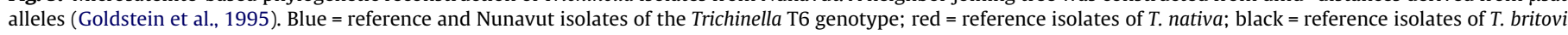

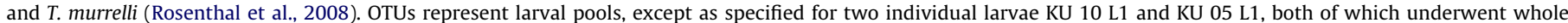
genome amplification prior to microsatellite genotyping. 


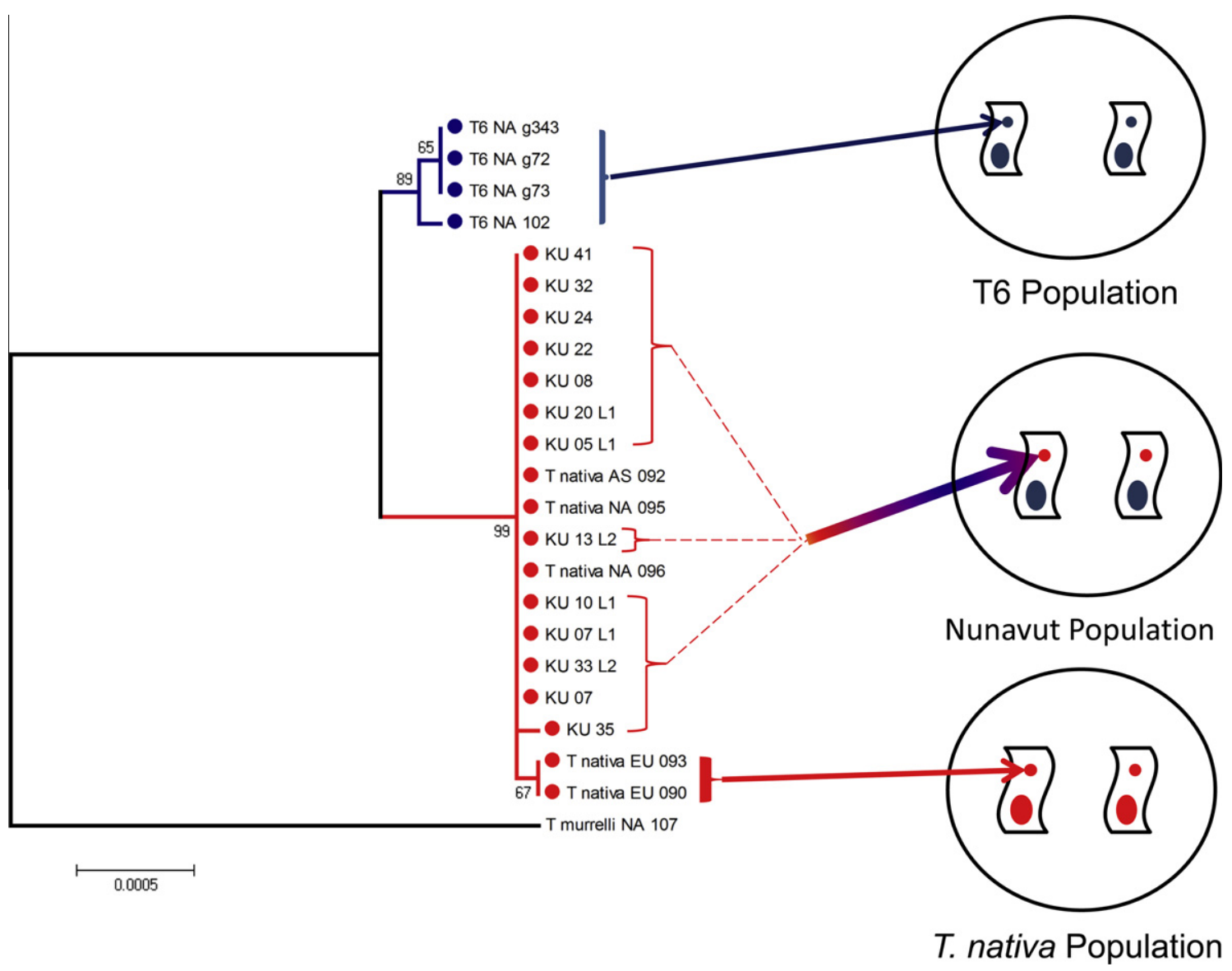

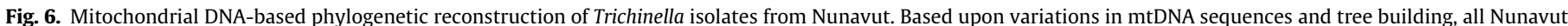


subpopulation of isolates to the Trichinella T6 genotype (see large arrow).

depicted in Fig. 6. Interestingly, although these data substantiate the survival of hybrids birthed from female T. nativa, the offspring from exactly such experimental crosses were found to be infertile in a previous study (La Rosa et al., 2003). Perhaps the natural situation arose when F1 hybrids back-crossed with parental types. Alternatively, the genetic differentiation between T. nativa and T6 may not, in nature, be as complete as in the several reference isolates examined to date.

Avise (2001) reviewed many cases of introgressive hybridization spanning a broad range of plant and animal species, reflecting interesting differences in the temporal and geographic scales. Genetic admixture may or may not be confined to very narrow borders of overlap between well-differentiated taxa; introgression may be reciprocal or highly biased (in the contribution of each lineage), and may derive either from occasional transient or ongoing processes. The extent of observed mosaicism in nuclear genomes provides a means to infer the consequences of such interbreeding; 'capture' of distinct organellar genomes (such as the mtDNA of $T$. nativa, in this instance) is a frequent outcome of introgression.

More intensive sampling in Nunavut might well uncover individuals whose nuclear genomes bear attributes of both lineages (schematically represented in scenario 3 of Fig. 1). We cannot conclude from the present sample whether hybridization is rare, whether poor survival or reproduction characterizes most progeny of such hybridizations, or whether hybridization has only recently commenced. Each might explain the persistence of distinctions between the nuclear genomes of T. nativa and T6. We may be witnessing introgression among populations once separated by glacial barriers; given the likelihood that these lineages diverged only recently (Zarlenga et al., 2006). It is also conceivable that shared polymorphisms are the consequence of incomplete sorting of shared ancestral variation. Resolving these alternatives would provide a rational basis for determining whether $\mathrm{T} 6$ warrants recognition as a distinct species, or simply as a genetic variant of $T$. nativa. Future analysis of gene flow between these two genotypes could be explored by richer sampling of additional populations of wild animals. Methods such as those used here, if applied on a broader geographic scale, could more definitively establish the origins and significance of the apparent distinction between T. nativa and T6.

\section{Acknowledgments}

Funding for wolverine sample collection and transport was provided by the Department of Environment, Government of Nunavut; the Nunavut Wildlife Management Board; and the Center for Veterinary Health Sciences, Oklahoma State University. We would like to thank the Kugluktuk Hunters and Trappers Organization and the participating hunters for their support of this research project, and two anonymous reviewers for their constructive criticisms.

\section{References}

Avise, J.C., 2001. Molecular Markers, Natural History, and Evolution. Klewar Academic Publishers, Norwell, MA.

Davidson, R.K., Handeland, K., Kapel, C.M., 2008. High tolerance to repeated cycles of freezing and thawing in different Trichinella nativa isolates. Parasitol. Res. 103 (5), 1005-1010.

Eaton, R.D., 1979. Trichinosis in the Arctic. Can. Med. Assoc. J. 120 (1), 22. 
Gajadhar, A.A., Forbes, L.B., 2010. A 10-year wildlife survey of 15 species of Canadian carnivores identifies new hosts or geographic locations for Trichinella genotypes T2, T4, T5, and T6. Vet. Parasitol. 168 (1-2), 78-83.

Goldstein, D.B., Ruiz Linares, A., Cavalli-Sforza, L.L., Feldman, M.W., 1995. An evaluation of genetic distances for use with microsatellite loci. Genetics 139 (1), 463-471.

Gottstein, B., Pozio, E., Nockler, K., 2009. Epidemiology, diagnosis, treatment, and control of trichinellosis. Clin. Microbiol. Rev. 22 (1), 127-145, Table of Contents.

Kapel, C.M., 1997. Trichinella in arctic, subarctic and temperate regions: Greenland, the Scandinavian countries and the Baltic States. Southeast Asian J. Trop. Med. Public Health 28 (Suppl. 1), 14-19.

Kapel, C.M., Henriksen, S.A., Berg, T.B., Nansen, P. 1995. Trichinella infections in arctic foxes from Greenland: studies and reflections on predilection sites of muscle larvae. J. Helminthol. 69 (4), 325-330.

Kapel, C.M., Pozio, E., Sacchi, L., Prestrud, P., 1999. Freeze tolerance, morphology, and RAPD-PCR identification of Trichinella nativa in naturally infected arctic foxes. J. Parasitol. 85 (1), 144-147.

Kumar, S., Nei, M., Dudley, J., Tamura, K., 2008. MEGA: a biologist-centric software for evolutionary analysis of DNA and protein sequences. Brief. Bioinform. 9 (4), 299-306.

La Rosa, G., Marucci, G., Zarlenga, D.S., Casulli, A., Zarnke, R.L., Pozio, E., 2003. Molecular identification of natural hybrids between Trichinella nativa and Trichinella T6 provides evidence of gene flow and ongoing genetic divergence. Int. J. Parasitol. 33 (2), 209-216.

La Rosa, et al., 2012. Development of a single larva microsatellite analysis to investigate the population structure of Trichinella spiralis' infection. Genet. Evol., in press.

Lavrov, D.V., Brown, W.M., 2001. Trichinella spiralis mtDNA: a nematode mitochondrial genome that encodes a putative ATP8 and normally structured tRNAs and has a gene arrangement relatable to those of coelomate metazoans. Genetics 157 (2), 621-637.

MacLean, J.D., Viallet, J., Law, C., Staudt, M., 1989. Trichinosis in the Canadian Arctic: report of five outbreaks and a new clinical syndrome. J. Infect. Dis. 160 (3), 513520.

MacLean, J.D., Poirier, L., Gyorkos, T.W., Proulx, J.F., Bourgeault, J., Corriveau, A., Illisituk, S., Staudt, M., 1992. Epidemiologic and serologic definition of primary and secondary trichinosis in the Arctic. J. Infect. Dis. 165 (5), 908-912.
Malakauskas, A., Kapel, C.M., 2003. Tolerance to low temperatures of domestic and sylvatic Trichinella spp. in rat muscle tissue. J. Parasitol. 89 (4), 744-748.

Pozio, E., Zarlenga, D.S., 2005. Recent advances on the taxonomy, systematics and epidemiology of Trichinella. Int. J. Parasitol. 35 (11-12), 1191-1204.

Pozio, E. La Rosa, G. Rossi, P., Murrell, K.D., 1992. Biological characterization of Trichinella isolates from various host species and geographical regions. J. Parasitol. 78 (4), 647-653.

Pozio, E., Hoberg, E., La Rosa, G., Zarlenga, D.S., 2009. Molecular taxonomy, phylogeny and biogeography of nematodes belonging to the Trichinella genus. Infect. Genet. Evol. 9 (4), 606-616.

Reichard, M.V., Torretti, L., Snider, T.A., Garvon, J.M., Marucci, G., Pozio, E., 2008 Trichinella T6 and Trichinella nativa in Wolverines (Gulo gulo) from Nunavut, Canada. Parasitol. Res. 103 (3), 657-661.

Rosenthal, B.M., LaRosa, G., Zarlenga, D., Dunams, D., Chunyu, Y., Mingyuan, L., Pozio, E., 2008. Human dispersal of Trichinella spiralis in domesticated pigs. Infect. Genet. Evol. 8 (6), 799-805.

Serhir, B., MacLean, J.D., Healey, S., Segal, B., Forbes, L., 2001. Outbreak of trichinellosis associated with arctic walruses in northern Canada, 1999. Can. Commun. Dis. Rep. 27 (4), 31-36.

Tamura, K., Dudley, J., Nei, M., Kumar, S., 2007. MEGA4: Molecular Evolutionary Genetics Analysis (MEGA) software version 4.0. Mol. Biol. Evol. 24 (8), 15961599.

Yang, B.Z., Zhao, H., Kranzler, H.R., Gelernter, J., 2005. Practical population group assignment with selected informative markers: characteristics and properties of Bayesian clustering via STRUCTURE. Genet. Epidemiol. 28 (4), 302-312.

Zarlenga, D.S., Chute, M.B., Martin, A., Kapel, C.M., 1999. A multiplex PCR for unequivocal differentiation of all encapsulated and non-encapsulated genotypes of Trichinella. Int. J. Parasitol. 29 (11), 1859-1867.

Zarlenga, D.S., Chute, M.B., Martin, A., Kapel, C.M., 2001. A single, multiplex PCR for differentiating all species of Trichinella. Parasite 8 (Suppl. 2), S24-26.

Zarlenga, D.S., Rosenthal, B.M., La Rosa, G., Pozio, E., Hoberg, E.P., 2006. PostMiocene expansion, colonization, and host switching drove speciation among extant nematodes of the archaic genus Trichinella. Proc. Natl. Acad. Sci. USA 103 (19), 7354-7359. 